\title{
Effect of electropolymerization potential on the preparation of PEDOT/graphene oxide hybrid material for supercapacitor application
}

\begin{abstract}
Conducting polymer poly(3,4-ethylenedioxythipohene) (PEDOT) hybrid with carbon-based material, graphene oxide (GO), was prepared for supercapacitor application. Different applied potentials were employed in order to study the effect of electropolymerization potential on PEDOT/GO thin film. Field emission scanning electron microscopy (FESEM) images showed that PEDOT/GO possessed more pronounced wrinkle paper-like sheet surface morphology as the potential increased from 1.0 to $2.0 \mathrm{~V}$. Fourier transform infrared spectroscopy (FTIR) and Raman spectroscopy revealed that GO was successfully incorporated into PEDOT during electropolymerization. The cyclic voltammetry (CV) and galvanostatic charge-discharge (GCD) measurements revealed that the PEDOT/GO composite electropolymerized at the applied potential of $1.2 \mathrm{~V}$ exhibited a maximum specific capacitance of $115.15 \mathrm{~F} / \mathrm{g}$ with energy density and power density of $13.60 \mathrm{Wh} / \mathrm{kg}$ and 139.09 $\mathrm{W} / \mathrm{kg}$, respectively at current density $0.3 \mathrm{~A} / \mathrm{g}$. The EIS result showed that the Rct decreased as the electropolymerization potential rose from $1 \mathrm{~V}$ to $1.2 \mathrm{~V}$ and increased when the electropolymerization further increased to $2 \mathrm{~V}$ due to a large electron transfer resistance that makes the rate of charge transfer becomes slower.
\end{abstract}

Keyword: Poly(3,4-ethylenedioxythipohene); Supercapacitor; Graphene oxide 